Revised Submission to Annals of Surgery

Article Title:

\title{
Could variations in technical skills acquisition in surgery be explained by differences in cortical plasticity?
}

\author{
Authors \\ Mr Daniel Richard Leff (MB BS MRCS), Mr Julian JH Leong (MA MRCS), \\ Mr Rajesh Aggarwal (MA MRCS), Professor Guang-Zhong Yang (PhD), \\ Professor Sir Ara Darzi (MD, FRCS, FACS, FMedSci) \\ Royal Wolfson Medical Image Computing Laboratory and \\ Department of BioSurgery and Surgical Technology \\ Imperial College London \\ United Kingdom
}

\section{Article Category:}

Brief Review Article

\section{Abstract Word Count:}

250

\section{Word Count:}

1,552

Financial Disclosure: None to declare

Conflict of Interest: The Royal Wolfson Medical Image Computing Laboratory and Department of BioSurgery and Surgical Technology at Imperial College London have recently purchased an Optical Topography device (ETG-4000, Hitachi Medical Corporation, Japan) to study cortical plasticity associated with technical skills acquisition in surgery 


\section{$\underline{\text { Revised Abstract }}$}

Variations in technical performance in surgery are known to exist but are poorly understood. Gaining an appreciation of these differences may have implications for technical skills training, assessment and selection. Investigators attempting to correlate technical skill with visuospatial or perceptual tests, have failed to identify surrogate markers of surgical aptitude. Evidence from unrelated fields suggests that studying brain function may advance our understanding of disparate technical performance in surgery. The brain is dynamic and patterns of activation vary with experience and training, a property referred to as 'neuroplasticity'. Functional neuroimaging studies of complex non-surgical skills have demonstrated smaller, more refined neuronal networks in experts compared to novices. Novel unrefined performance places a significant burden on generic areas of attention and control such as the anterior cingulate cortex (ACC) and the prefrontal cortex (PFC). These regions are recruited less as skills are performed with increasing automaticity. Persistent PFC activation has been shown to herald poor bimanual co-ordination learning in studies involving non-surgical tasks. It is hypothesised that alterations in brain activation foci accompany a transition through phases of surgical skills learning and that those patterns of activation may vary according to technical ability. Validating this hypothesis is challenging, since requires studying brain function in ambulant subjects performing complex motor skills. In a surgical knot-tying study involving over sixty subjects of varying expertise, PFC activation was identified in novices but not in trained surgeons. Further work should aim to determine if PFC activation attenuate in the context of learning success in surgery. 
$\underline{\text { Revised Submission to Annals of Surgery }}$

Article Title:

Could variations in technical skills acquisition in surgery be explained by differences in cortical plasticity?

\author{
$\underline{\text { Authors }}$ \\ Mr Daniel Richard Leff (MB BS MRCS), Mr Julian JH Leong (MA MRCS), \\ Mr Rajesh Aggarwal (MA MRCS), Professor Guang-Zhong Yang (PhD), \\ Professor Sir Ara Darzi (MD, FRCS, FACS, FMedSci) \\ Royal Wolfson Medical Image Computing Laboratory and \\ Department of BioSurgery and Surgical Technology \\ Imperial College London \\ United Kingdom
}

\title{
Article Category:
}

Brief Review Article

\section{Abstract Word Count:}

250

\section{Word Count:}

1,552

Financial Disclosure: None to declare

Conflict of Interest: The Royal Wolfson Medical Image Computing Laboratory and Department of BioSurgery and Surgical Technology at Imperial College London have recently purchased an Optical Topography device (ETG-4000, Hitachi Medical Corporation, Japan) to study cortical plasticity associated with technical skills acquisition in surgery 


\section{$\underline{\text { Revised Abstract }}$}

Variations in technical performance in surgery are known to exist but are poorly understood. Gaining an appreciation of these differences may have implications for technical skills training, assessment and selection. Investigators attempting to correlate technical skill with visuospatial or perceptual tests, have failed to identify surrogate markers of surgical aptitude. Evidence from unrelated fields suggests that studying brain function may advance our understanding of disparate technical performance in surgery. The brain is dynamic and patterns of activation vary with experience and training, a property referred to as 'neuroplasticity'. Functional neuroimaging studies of complex non-surgical skills have demonstrated smaller, more refined neuronal networks in experts compared to novices. Novel unrefined performance places a significant burden on generic areas of attention and control such as the anterior cingulate cortex (ACC) and the prefrontal cortex (PFC). These regions are recruited less as skills are performed with increasing automaticity. Persistent PFC activation has been shown to herald poor bimanual co-ordination learning in studies involving non-surgical tasks. It is hypothesised that alterations in brain activation foci accompany a transition through phases of surgical skills learning and that those patterns of activation may vary according to technical ability. Validating this hypothesis is challenging, since requires studying brain function in ambulant subjects performing complex motor skills. In a surgical knot-tying study involving over sixty subjects of varying expertise, PFC activation was identified in novices but not in trained surgeons. Further work should aim to determine if PFC activation attenuate in the context of learning success in surgery. 


\section{Main Manuscript}

\section{Background}

Technical skill is an important attribute of any aspiring surgeon. The technically astute are likely to have better mortality and morbidity outcomes, a valuable asset in the face of an ever more knowledgeable public. Objective assessments tools have demonstrated a range of technical abilities amongst surgeons with parallel operative experience, as well as overlapping performance between surgeons of differing grades

1,2. Residents acquire skills at different rates and some may even be unable to achieve a stable task performance ${ }^{3}$. The reasons why certain residents rapidly progress whilst others struggle despite similar training environments remains a mystery. Shedding light on these differences may have significant implications for surgical training and selection.

Recognition of poor progress may enable interventions that improve technical skills training. Moreover, early identification of technically competent surgical interns might assist trainers in the selection of future surgeons. Interestingly, on both sides of the Atlantic, surgical selection procedures remain highly controversial ${ }^{4}$ and manual skill is rarely used as a criterion. Evidence suggests that in the United States (US), technical skills evaluation is performed as a selection criterion in only $5 \%$ of programs ${ }^{5}$. In the United Kingdom (UK), despite recent drastic reforms to selection procedures, at no point are technical skills evaluated. Consequently, there are few reports evaluating the relationship between manual skill at selection and overall performance in residency programs. Instead, investigators have attempted to explore surrogate markers of technical skills, in the hope of improving surgical selection if strong correlations could be identified. 
Theoretically, more efficient individuals may have an innate advantage or 'aptitude' over poorly performing subjects. Investigators have attempted to correlate technical performance with handedness, sex, intelligence, as well as innate visuomotor and perceptual ability. However, these attributes have tended to correlate poorly with technical skill in surgery ${ }^{6}$. Consultants have performed worse than residents in tests of pure manual dexterity ${ }^{7}$. With a few notable exceptions ${ }^{8}$ tests of visuospatial and perceptual ability have failed to be indicators of surgical prowess ${ }^{6}$. Even where correlations between abstract tests and technical skills have been demonstrated ${ }^{9}$, they have often relied upon poor indicators of surgical quality, such as task completion times.

\section{Motor skills learning at cortical level}

How then to rationalise the disparity in technical skills acquisition in surgery to improve training and selection? It is suspected that differences in innate motor learning capability may account for the diversity in technical skill. Whilst educators have made considerable developments to objectify assessment of surgical skills using methods such as dexterity analysis and video based ratings of performance; neuroanatomists, neurophysiologists and behavioural psychologists have made similar advances to the understanding of motor skills learning at cortical level. Consummate motor performance relies upon a distributed network of cortical and subcortical brain activation. The brain is dynamic and activation foci vary according to the stage or phase of leaning, a property referred to as 'neuroplasticity' ${ }^{10}$. There is increasing evidence to suggest that subjects who rapidly assimilate motor tasks (non-surgical) have different patterns of plasticity from those who find technical skills challenging ${ }^{11 \text {, }}$ 
12. Motor learning capability can be inferred from brain activation quantified using non-invasive technologies.

\section{The role of the prefrontal cortex in novel task performance}

The hallmark of learning a new complex motor skill such as playing the piano, riding a bike or even learning to tie a surgical reef knot is that one progresses from an initial stage that is highly attention demanding to an advanced stage whereby skills run off automatically. Many surgical maneouvres entail chaining together components of prelearned sequences into new spatio-temporal arrangements, to produce smooth bimanual co-ordination. Studies of non-surgical bimanual motor sequence learning suggest that the prefrontal cortex (PFC) is vital during novel performance and redundant when tasks are executed with greater automaticity ${ }^{13-15}$. Improvements in the initial phases of motor skill learning rely upon correct response selection from visuospatial working memory, attention to action, error detection and recovery of attentional control in response to errors, all of which are subserved by the PFC ${ }^{16-19}$. The difficulty experienced by patients with PFC lesions in learning novel motor tasks is testimony that this region is vital for technical skills acquisition ${ }^{20}$. Generic attention and control areas such as the PFC are thought to act as 'scaffold' to support unrefined performance ${ }^{21}$. Eventually, as skills are honed through practice, the scaffold is no longer necessary and falls away.

Could PFC activation be important in the preliminary phases of technical skills acquisition in surgery? Attenuation in PFC activation might be anticipated in the context of learning success. Similarly, persistent PFC activation may herald learners in need of remedial training. Residents still having to ruminate the rudimentary tasks 
such as tying a surgical reef knot, for example, are unlikely to gain from other learning experiences within the operating theatre ${ }^{22}$. A recent evaluation of nonsurgical knot tying demonstrated preferential activation in anterior brain regions such as the PFC in subjects unable to grasp this motor skill ${ }^{11}$. In contrast, activation was rapidly relegated to posterior structures in 'strong learners', possibly reflecting a shift in cognitive resources indicative of a more passive mode of attention ${ }^{11}$.

\section{Cortical plasticity associated with phases of motor skill learning}

Reznick et al ${ }^{22}$ recently suggested that technical skills training should follow established motor learning theory ${ }^{22}$. Residents should practice in safe environments until skills progress from a highly attention demanding 'cognitive' phase where error detection confers performance gains to a phase in which tasks are performed in an 'aysmptotic' manner. Evaluating cortical function in conjunction with performance metrics may help to track a trainee's progression through these phases of learning. Practice dependent plasticity, can be measured as changes in cortical activation maps. Motor skills training leads to a gradually evolving more extensive representation of the trained sequence in the primary motor cortex (M1), a so-called 'slow learning' effect $^{23}$. Similar plasticity in M1 has been observed for acquisition of visuomotor sequences ${ }^{24}$ and tasks requiring bimanual co-ordination ${ }^{15}$ in healthy humans as well as in non-human primates ${ }^{25}$.

Ultimately, well-learned motor routines are resistant to interference and the passage of time and can be readily retrieved with reasonable performance despite long periods without practice. Automatisation leads to decreased activation in several 
supplementary motor regions outside of $\mathrm{M} 1{ }^{15}$. We hypothesise that similar synaptic modifications occur during technical skills training in surgery.

\section{The effects of long term training and deliberate practice}

Musicians have offered an opportunity to explore the effects of daily practice upon the adaptive plasticity of the sensorimotor system. Structural ${ }^{26,27}$ and functional adaptation ${ }^{28}$ occurs in response to intensive musical training. Increases in the size of the corpus callosum ${ }^{27}$, the hand motor area ${ }^{26}$ and structural adaptation of the cerebellum ${ }^{29}$ have all been observed in professional musicians. Moreover, during execution of visuomotor sequences and finger to thumb opposition tasks, musically naïve subjects activate a wider network of cortical and subcortical brain regions and with greater intensity than do their professional musical counterparts ${ }^{28,30}$. Skilled musicians seem to activate smaller neural networks, thereby controlling bimanual movement in a more efficient manner. It is conceivable that through daily practice, expert surgeons may harness similar neuronal efficiency. Practice related improvements in technical performance such as those demonstrated using dexterity analysis systems (reduced number of movements to complete a task, etc) are likely to be due greater cerebral efficiency. Theoretically, more extensive activations and upregulation of supplementary motor regions might be anticipated in novices and /or in poorly performing surgeons.

\section{Concluding Hypothesis}

It is apparent that cortical modifications occur in response to complex motor skills learning. What we do not know is whether similar functional transformations occur in the brain of surgical residents as they learn to assimilate surgical procedures. Our 
hypothesis is that neuroplasticity is likely to accompany expertise development in surgery. Indeed, the 'surgical brain' may prove an intriguing model for studying plasticity owing to the complexity of the stimulus - surgery, and the duration of exposure to the stimulus ${ }^{31}$.

Elucidating the neurocognitive mechanisms that underpin surgical learning may assist training and selection of future surgeons. However, evaluating cortical function in ambulant subjects performing complex surgical tasks represents a significant methodological challenge. Indirect neuroimaging techniques that monitor the haemodynamic response, such as functional magnetic resonance imaging (fMRI), have environmental and practical constraints. Imaging methods that directly measure neuronal activation, such as electroencephalography (EEG), are comparatively poor at source localisation. Our initial efforts have focused on using Optical Topography (OT), a technique that employs near infrared spectroscopy to quantify the haemodynamic response to cortical activation. Unlike other imaging techniques, OT is discrete, portable, cost-effective, and tolerant to body motion, enabling evaluation in realistic environments. In a block design experiment of surgical knot-tying involving sixty-two subjects of varying surgical expertise, prefrontal activation was isolated in surgical novices but not in expert surgeons ${ }^{32}$. Figure 1 (panels a and b) illustrates the OT experimental set up and provides an example of cortical oxygenation change for a typical expert and novice subject. Further work is required to determine if prefrontal activation wanes in the context of learning success in surgery. 
Our hypothesis is that disparity in technical performance and acquisition of surgical skills may be explained by differences in motor learning capability, indexed by cortical function and neuroplasticity. Exposing these mechanisms requires a multimodal strategy involving pervasive measurements of dexterity, visual attention and brain function. These methods may assist the identification of learners in need of assistance and selection of future surgeons based upon desirable attributes. 


\section{$\underline{\text { References }}$}

1. Datta V, Chang A, Mackay S, Darzi A. The relationship between motion analysis and surgical technical assessments. Am J Surg 2002; 184:70-3.

2. Grantcharov TP, Bardram L, Funch-Jensen P, Rosenberg J. Learning curves and impact of previous operative experience on performance on a virtual reality simulator to test laparoscopic surgical skills. Am J Surg 2003; 185:1469.

3. Schijven MP, Jakimowicz J. The learning curve on the Xitact LS 500 laparoscopy simulator: profiles of performance. Surg Endosc 2004; 18:121-7.

4. MMC: mass medical culling. Lancet 2007; 369:879.

5. Bernstein AD, Jazrawi LM, Elbeshbeshy B, Della Valle CJ, Zuckerman JD. An analysis of orthopaedic residency selection criteria. Bull Hosp Jt Dis. 2002-2003; 61:49-56

6. Anastakis DJ, Hamstra SJ, Matsumoto ED. Visual-spatial abilities in surgical training. Am J Surg 2000; 179:469-71.

7. Squire D, Giachino AA, Profitt AW, Heaney C. Objective comparison of manual dexterity in physicians and surgeons. Can J Surg 1989; 32:467-70.

8. Wanzel KR, Hamstra SJ, Anastakis DJ, et al. Effect of visual-spatial ability on learning of spatially-complex surgical skills. Lancet 2002; 359:230-1.

9. Risucci D, Geiss A, Gellman L, et al. Surgeon-specific factors in the acquisition of laparoscopic surgical skills. Am J Surg 2001; 181:289-93.

10. Doyon J, Benali H. Reorganization and plasticity in the adult brain during learning of motor skills. Curr Opin Neurobiol 2005; 15:161-7.

11. Tracy J, Flanders A, Madi S, et al. Regional brain activation associated with different performance patterns during learning of a complex motor skill. Cereb Cortex 2003; 13(9):904-10.

12. Haier RJ, Siegel BV, Jr., MacLachlan A, et al. Regional glucose metabolic changes after learning a complex visuospatial/motor task: a positron emission tomographic study. Brain Res 1992; 570:134-43.

13. Inoue K, Kawashima R, Satoh K, et al. A PET study of visuomotor learning under optical rotation. NeuroImage 2000; 11:505-16.

14. Debaere F, Wenderoth N, Sunaert S, et al. Changes in brain activation during the acquisition of a new bimanual coodination task. Neuropsychologia 2004; 42:855-67. 
15. Puttemans V, Wenderoth N, Swinnen SP. Changes in brain activation during the acquisition of a multifrequency bimanual coordination task: from the cognitive stage to advanced levels of automaticity. J Neurosci 2005; 25:42708.

16. Rowe J, Friston K, Frackowiak R, Passingham R. Attention to action: specific modulation of corticocortical interactions in humans. NeuroImage 2002; 17:988-98.

17. Baddeley A. Working memory. Oxford:: Oxford University Press, 1986.

18. Sarter M, Gehring WJ, Kozak R. More attention must be paid: the neurobiology of attentional effort. Brain Res Brain Res Rev 2006; 51:145-60.

19. Carter CS, Braver TS, Barch DM, et al. Anterior cingulate cortex, error detection, and the online monitoring of performance. Science 1998; 280:7479 .

20. Richer F, Chouinard MJ, Rouleau I. Frontal lesions impair the attentional control of movements during motor learning. Neuropsychologia 1999; $37: 1427-35$.

21. Petersen SE, van Mier H, Fiez JA, Raichle ME. The effects of practice on the functional anatomy of task performance. Proc Natl Acad Sci U S A 1998; 95:853-60.

22. Reznick RK, MacRae H. Teaching surgical skills--changes in the wind. $\mathrm{N}$ Engl J Med 2006; 355:2664-9.

23. Karni A, Meyer G, Rey-Hipolito C, et al. The acquisition of skilled motor performance: fast and slow experience-driven changes in primary motor cortex. Proc Natl Acad Sci U S A 1998; 95:861-8.

24. Floyer-Lea A, Matthews PM. Changing brain networks for visuomotor control with increased movement automaticity. J Neurophysiol 2004; 92:2405-12.

25. Nudo RJ, Milliken GW, Jenkins WM, Merzenich MM. Use-dependent alterations of movement representations in primary motor cortex of adult squirrel monkeys. J Neurosci 1996; 16:785-807.

26. Amunts K, Schlaug G, Jancke L, Steinmetz H, Schleicher A, Dabringhaus, A ZK. Motor cortex and hand motor skills: structural compliance in the human brain. Hum Brain Mapp 1997; 5:206 -215.

27. Schlaug G, Jancke L, Huang Y, et al. Increased corpus callosum size in musicians. Neuropsychologia 1995; 33:1047-55.

28. Krings $\mathrm{T}$, Topper $\mathrm{R}$, Foltys $\mathrm{H}$, et al. Cortical activation patterns during complex motor tasks in piano players and control subjects. A functional magnetic resonance imaging study. Neurosci Lett 2000; 278:189-93. 
29. Schlaug G. The brain of musicians. A model for functional and structural adaptation. Ann N Y Acad Sci 2001; 930:281-99.

30. Koeneke S, Lutz K, Wustenberg T, Jancke L. Long-term training affects cerebellar processing in skilled keyboard players. Neuroreport 2004; 15:127982 .

31. Munte TF, Altenmuller E, Jancke L. The musician's brain as a model of neuroplasticity. Nat Rev Neurosci 2002; 3:473-8.

32. Leff DR, Leong JJH, Atallah L, Koh PH, Elwell C, Delpy DT, Yang GZ, Darzi AW. Open Surgical Knot-Tying Induces a Lateralised Prefrontal Brain Response in Surgical Novices. 13th Annual Meeting of the Organization for Human Brain Mapping. NeuroImage (2007). 


\section{Figure Legends}

\section{Figure 1 (panel a)}

Schematic illustration of Optical Topography optode placement. Two $3 \times 3 \mathrm{~cm}$ optode probe arrays were used. For each probe array, light emitters (red) and detectors (blue) were positioned according to the 10-20 system for electrode placement. This arrangement provided 24 measuring positions or 'channels' (grey shaded boxes). Channels 1-12 were positioned on the right prefrontal cortex and channels 13-24 mirrored these positions on the left prefrontal cortex. Knot-tying dexterity was quantified using the Imperial College Surgical Assessment Device (ICSAD). ICSAD motion tracker sensors are worn on the dorsum of each hand as illustrated. 


\section{Figure 1 (panel b)}

Dynamic topographic maps of cortical oxyhaemoglobin intensity change observed in a typical novice (top row) and expert surgeon (bottom row). Columns represent baseline rest, 2, 6 and 10 seconds into a surgical knot-tying trial. Emitters (red circles), detectors (blue circles) and corresponding channels (pink circles) are overlaid onto high resolution T1 weighted MRI images. (Republished with permission from Springer). 
Acknowledgements: The authors would like to thank all the surgeons and medical students that participated. We extend our thanks to Dr Alex Hammers for his expertise with MRI overlay and Mr James Kinross for his help with illustrations. 


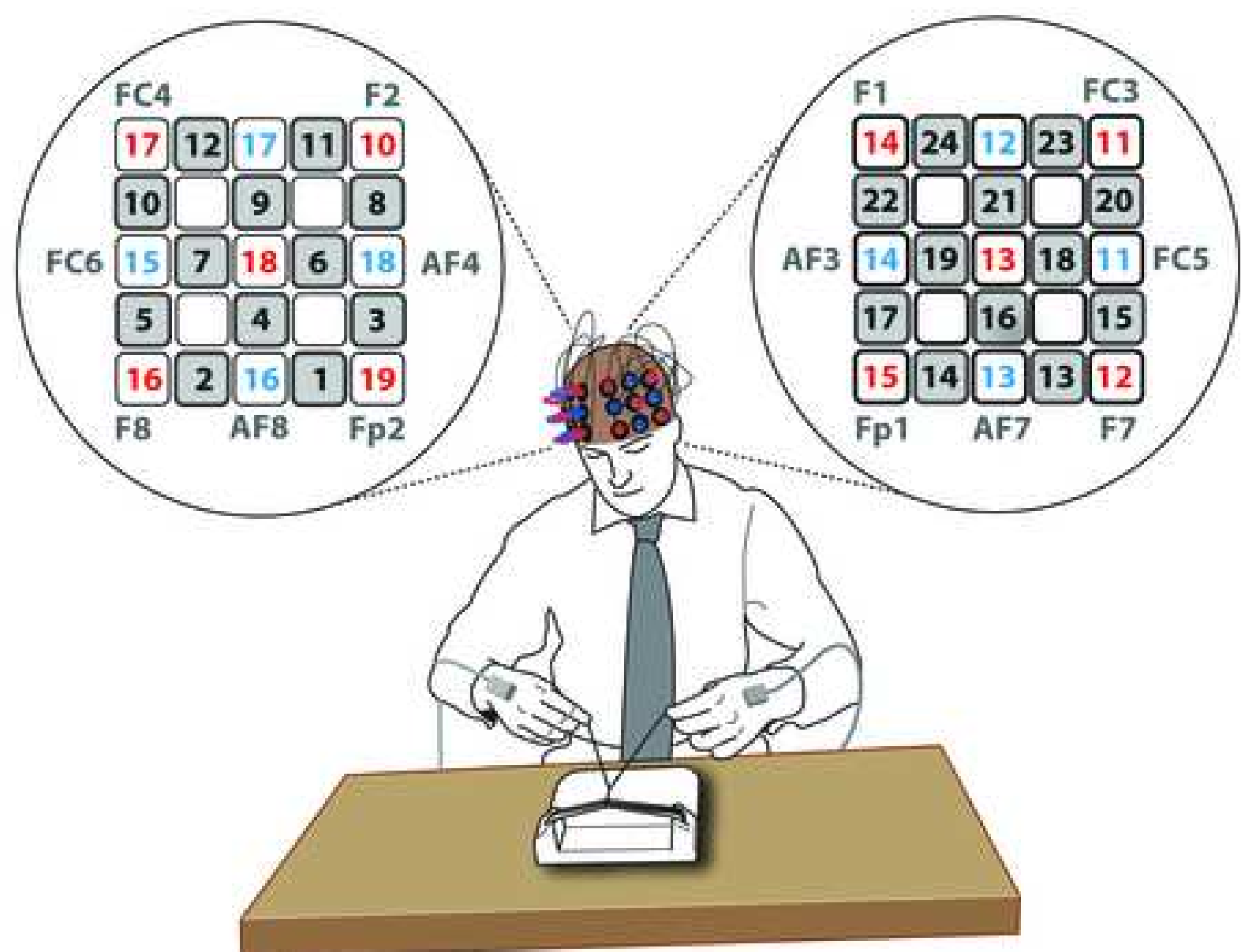



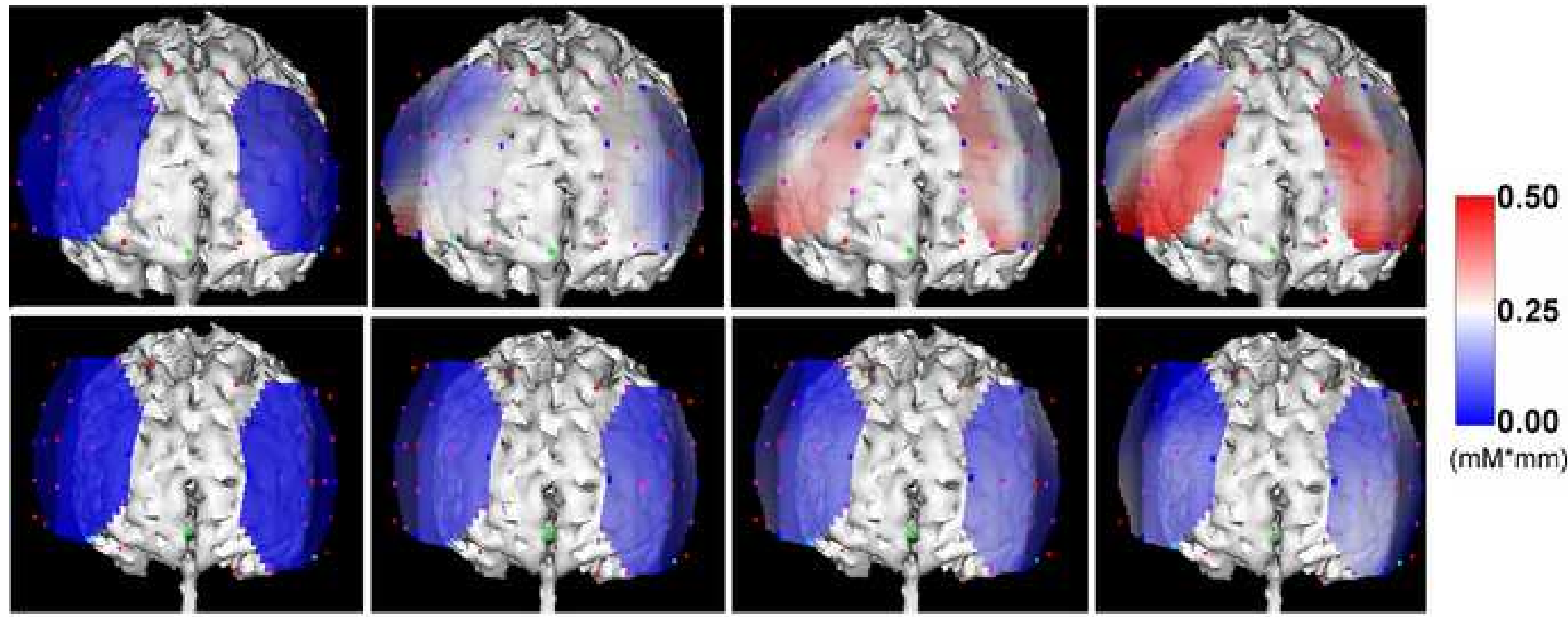

$(\mathrm{mM} \cdot \mathrm{mm})$ 


\title{
${ }^{*}$ Transfer of Copyright
}

This piece of the submission is being sent via mail.

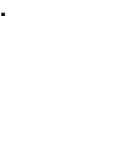

\begin{abstract}
Transfer of Copyrighn
\end{abstract}

(1)

ardor

(⿸丆口

.

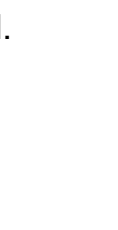

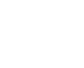

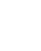

